Pacific Journal of Mathematics

INCOMPRESSIBILITY OF MAPS AND THE HOMOTOPY 


\title{
INCOMPRESSIBILITY OF MAPS AND THE HOMOTOPY INVARIANCE OF ČECH COHOMOLOGY
}

\author{
Allan Calder aNd Frank Williams
}

The $\Omega$-compressibility dimension of a space $Y$ is the largest integer $r$ for which every map $f: X \rightarrow Y$ from a normal space with dimension less than $r$, the loop map $\Omega f: \Omega X \rightarrow \Omega Y$ is compressible. Bounds are determined for the $\Omega$-compressibility dimension of Eilenberg-Maclane spaces of type $(Z, 2 n)$ and $\left(Z_{p} k, 2 n\right)$. In application this is used to settle the question as to when Cech cohomology based on finite covers is a homotopy invariant functor.

1. Compressibility: Background and statement results. A map is called compressible if it is homotopic to a map whose image is contained in a compact subset of the target space. Let $\Omega$ denote the loop functor. A map $f: X \rightarrow Y$ is called $\Omega$-compressible if $\Omega f$ : $\Omega X \rightarrow \Omega Y$ is compressible.

Obviously if $\Omega Y$ has the homotopy type of a compact space then any map into $Y$ is $\Omega$-compressible. On the other hand if $\Omega Y$ has nonzero homology (or homotopy) groups in infinitely many dimensions then it is easy to see that the identity map $1_{Y}: Y \rightarrow Y$ is $\Omega$-incompressible. In particular that will be the case when $Y$ is a finite complex with nonzero (reduced) homology.

We shall be primarily concerned with the case $Y=K(G, n)$, an Eilenberg-Maclane space. Any essential map $f: S^{2 n+1} \rightarrow K(Z, 2 n+1)$ is $\Omega$-incompressible since $\Omega f$ induces an isomorphism,

$$
(\Omega f)^{*}: H^{*}(\Omega K(Z, 2 n+1) ; Q) \longrightarrow H^{*}\left(\Omega S^{2 n+1} ; Q\right),
$$

in rational cohomology. The following fundamental result is due to Weingram [11] (see also [9]).

WEINGRAM's THEOREM. For any finitely generated abelian group $G$, every essential map $f: S^{2 n+1} \rightarrow K(G, 2 n+1)$ is $\Omega$-incompressible.

For even dimensional spheres the situation is more complicated: if $p$ is an odd prime then any map $S^{2 n} \rightarrow K\left(Z_{p} k, 2 n\right)$ is $\Omega$-compressible, since $S^{2 n-1}$ is a $H$-space $\bmod p$ (see [9] for details). Similarly, if $n=1,2$ or 4 then any $\operatorname{map} S^{2 n} \rightarrow K(G, 2 n)$ is $\Omega$-compressible for any $G$.

Any map from an $n$-dimensional (covering dimension) normal space into a CW-complex can be homotopically deformed into a map whose image is in the $n$-skeleton of the target space [see e.g., $6 \mathrm{p}$. 
356]. The $n$-skelton of $K(Z, n)$ (or $K\left(Z_{p} k, n\right)$ ) can be considered to be $S^{n}$. So if the inclusion of $S^{n}$ is $\Omega$-compressible then so is every map from any $n$-dimensional space into $K(Z, n)$ (or $K\left(Z_{p} k, n\right)$ ). When this is the case, it seems reasonable to ask what the dimension of the domain must be in order that there be an $\Omega$-incompressible map into $K(Z, n)$ (or $K\left(Z_{p} k, n\right)$ ).

DeFINITION 1.1. The $\Omega$-compressibility dimension of a space $Y$, denoted by $c(Y)$, is the greatest integer $r$ such that if $X$ is a normal space of dimension less than $r$ then every map $X \rightarrow Y$ is $\Omega$-compressible. If no such greatest integer exists we set $c(Y)=\infty$.

If $Y=K(G, n)$, we denote $c(Y)$ by $c(G, n)$.

EXAMPLE 1.2. From preceding remarks, we have:

(1) $c(Y)=\infty$ if $\Omega Y$ is homotopic to a compact space. Thus $c(Z, 2)=\infty$.

(2) $c(Z, 2 n+1)=c\left(Z_{p} k, 2 n+1\right)=2 n+1$.

(3) $c\left(Z_{p} k, 2 n\right)>2 n$ if $n \geqq 1$ and $p$ is odd.

(4) $c(G, 2 n)>2 n$ if $n=1,2$ or 4 .

In this paper we show that $c(Z, 2 n)$ and $c\left(Z_{p} k, 2 n\right)$ are finite for most values of $n$. Specifically in $\S 3$ we prove:

THEOREM 1.3. If $n \geqq 1, c\left(Z_{p} k, 2 n\right) \leqq 2 n+p-1$.

THEOREM 1.4. If $n \geqq 2, c(Z, 2 n) \leqq 2 n+9$.

We also wish to consider the concept analogous to $c(G, n)$ when $G=Q$. Since $K(Q, n)$ is not of finite type, we define $c^{*}(Y)$ in the same way as $c(Y)$ but restricting the domain spaces $X$ to be compact. Thus if $G$ is finitely generated, $c^{*}(G, n)=c(G, n)$. The following table summarizes the known results for $c(G, n)$ and $c^{*}(G, n)$ for $G=$ $Z_{p} k, Z$ and $Q$.

TABLE 1

\begin{tabular}{|c|c|c|c|c|}
\hline & \multicolumn{3}{|c|}{$c(G, n)=c^{*}(G, n)$} & $c^{*}(G, n)$ \\
\hline$n$ & $Z_{2} k$ & $Z_{p} k(p \neq 2)$ & $Z$ & $Q$ \\
\hline 1 & $\infty$ & $\infty$ & 1 & 1 \\
\hline odd $>1$ & $n$ & $n$ & $n$ & $n$ \\
\hline \multirow{3}{*}{$\begin{array}{c}4,8,16 \\
\text { other even }\end{array}$} & \multirow[b]{2}{*}{$n<c \leqq n+3$} & \multirow{3}{*}{$n<c \leqq n+2 p-1$} & $\infty$ & \multirow{3}{*}{$\infty$} \\
\hline & & & $\begin{aligned} n \leqq & \\
& \leqq n+9\end{aligned}$ & \\
\hline & $n \leqq c \leqq n+3$ & & & \\
\hline
\end{tabular}


The entries in Table 1 come from 1.2, 1.3, 1.4, and Table 2 in the next section.

It is still an open question as to what the exact values of $c(G, n)$ are in the last line of the table.

2. Čech cohomology: Background and statement of results. In 1932 Čech defined cohomology groups for an arbitrary space $X$ using the system of nerves of finite covers of $X,[5]$. These groups denoted by $H_{f}^{*}(X)$, have been ignored in favor of those based on arbitrary or locally finite covers, since Dowker showed that $H_{f}^{1}(X)$ is not a homotopy invariant of the space $X$, [7]. However it was subsequently shown in [1] that if $k>1$ and $G$ is finitely generated then $H_{f}^{k}(-; G)$ is a homotopy invariant functor on the category of finite dimensional normal spaces.

The homotopy invariance of $H_{f}^{k}(-; G)$ for larger categories was investigated in [4] using category-theoretic methods. Of primary importance there was the following theorem, in which $\beta$ denotes the Stone-Čech functor.

THEOREM 2.1. On the category of completely regular Hausdorff spaces the functors $H_{f}^{k}(-; G)$ and $[\beta-, K(G, k)]$ are naturally isomorphic.

\section{Proof. By [8] p. 282 and [6] p. 366.}

This theorem was used to apply results from [2,3] concerning the homotopy invariance of functors of the form $[\beta-, Y]$, to $H_{f}^{k}(-; G)$. We summarize the known results in the following table, which holds when $C$ is any full subcategory of the category of completely regular Hausdorff spaces that contains all countable CW-complexes.

TABLE 2. Is $H_{f}^{n}(-; G)$ a homotopy functor on $C$ ?

\begin{tabular}{c|c|c|c}
\hline$n-G=$ & $Z_{p} k$ & $Z$ & $Q$ \\
\hline 1 & YES & NO & NO \\
\hline 2 & & YES & YES \\
\hline $2 l+1(l \geqq 1)$ & NO & NO & NO \\
\hline $2 l(l>1)$ & & & YES \\
\hline
\end{tabular}

In $\S 4$ we will complete the table by proving the following theorem.

THEOREM 2.2. The functors $H^{2 n}\left(-; Z_{p} k\right)$ for $n \geqq 1$ and $H^{2 n}(-; Z)$ for $n>1$ are not homotopy invariant. 
Thus the blanks in the table can be filled in with "NO's".

3. Proofs of Theorems 1.3 and 1.4. The proofs of the theorems will be applications of a basic technical result, 3.1, the method of proof of which is essentially due to Goldfeather [9]. We attempt to follow Goldfeather's proof as closely as possible so as to make it clear where our arguments extend his and we include complete details, as the subtle nature of incompressibility seems to require fastidious exposition.

By a divided polynomial algebra over $Z$ we shall mean a free graded abelian group with generators $x_{1}, x_{2}, \cdots$ such that the algebra structure is given by $\left(x_{1}\right)^{i}=(i !) x_{i}$.

Let $\rho_{p}: H^{*}(X ; Z) \rightarrow H^{*}\left(X ; Z_{p}\right)$ be reduction $\bmod p$.

Theorem 3.1. Let $f: X \rightarrow Y$. Suppose that there exists $x \in$ $H^{*}(\Omega X ; Z)$ such that

(a) $x$ generates a divided polynomial algebra that is a summand of $H^{*}(\Omega X ; Z)$;

(b) $\rho_{p}(x) \in \operatorname{Im}\left\{(\Omega f)^{*}: H^{*}(\Omega Y ; Z) \rightarrow H^{*}\left(\Omega X ; Z_{p}\right)\right\}$; and

(c) $\tilde{H}^{*}(X ; Q)=0$ for $k \neq \operatorname{deg}(x)+1$.

Then $f$ is $\Omega$-incompressible.

Proof. Let $N=$ degree of $x$. By hypothesis there exist:

(1) $\chi \in H^{*}\left(\Omega Y ; Z_{p}\right)$ such that $(\Omega f)^{*}(\chi)=\rho_{p}(x)$;

(2) $\eta: \Omega Y \rightarrow K\left(Z_{p}, N\right)$ given by $\eta^{*}\left(\iota_{N}\right)=\chi$; and

(3) a fibration $g: \Omega X \rightarrow K\left(Z_{p}, N\right)$ given by $g^{*}\left(\iota_{N}\right)=\rho_{p}(x)$ where $\iota_{N}$ denotes a generator of $H^{N}\left(K\left(Z_{p}, N\right)\right)$.

Let $F$ denote the fiber of $g$ and consider the commutative diagram;

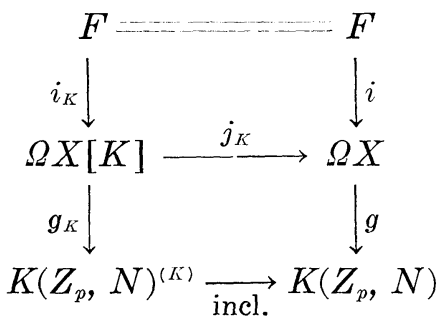

where $\Omega X[K]=g^{-1}\left(K\left(Z_{p}, N\right)^{(K)}\right)$, the portion of $\Omega X$ lying over the $K$-skeleton of $K\left(Z_{p}, n\right)$.

Suppose now that the divided polynomial algebra generated by $x$ is given by elements $x_{k} \in H^{k N}(\Omega X ; Z), k=1,2, \cdots$, such that $x^{k}=k ! x_{k}$.

Since $\rho_{p} i^{*}(x)=i^{*}\left(\rho_{p}(x)\right)=i^{*} g^{*}\left(\iota_{N}\right)=0$, and since $i^{*}: H^{N}(\Omega X ; Z) \rightarrow$ $H^{\wedge}(F ; Z)$ is a monomorphism (by the Serre exact sequence) we see that $i^{*}(x)$ is a nonzero of element of $H^{N}(F ; Z)$ that is divisible by 
p. Let $y$ be an element such that $i^{*}(x)=p y$. Now

so that

$$
i^{*}\left(x^{k}\right)=p^{k} y^{k},
$$

$$
i^{*}\left(x_{k}\right)=\frac{1}{k !} p^{k} y^{k} .
$$

Let $\sigma_{k}$ be the highest power of $p$ that divides $i^{*}\left(x_{k}\right)$ and $y_{k}$ be such that $i^{*}\left(x_{k}\right)=p^{\sigma_{k}} y_{k}$. Note that $\lim _{k} \sup \left(\sigma_{k}\right)=\infty$, since $(1 / k !) p^{k}$ is divisible by arbitrarily high powers of $p$.

Lemma 3.2. For each integer $K \geqq 0$ there exists an integer $\gamma(K)$ such that

$$
p^{\gamma(K)} H^{*}(F ; Z) \subset \operatorname{Im}\left(i_{K}^{*}\right) .
$$

Proof. Let $\mu(l)$ be the exponent of $p$ in $H^{l}\left(K\left(Z_{p}, N\right) ; Z\right)$. By the Universal Coefficient Theorem, the exponent of $p$ in $E_{2}^{l, *}$ in the Serre spectral sequence for $g$ is less than or equal to

$$
\mu^{\prime}(l)=\max (\mu(l), \mu(l+1)) .
$$

Let $\gamma(K)=\sum_{l=1}^{K} \mu^{\prime}(l)$. Then the group $p^{\gamma(K)} H^{*}(F ; Z)=p^{\gamma(K)} E_{2}^{0, *}$ is in the kernel of all the differentials $d_{r}$ for $2 \leqq r \leqq K$. Let $\left(E_{r}(K), d_{r}(K)\right)$ denote the spectral sequence of the fibration $g_{K}$. Since $E_{2}^{l, *}(K)=0$ for $l>K, d_{l}(K)=0$ for $l>K$. By naturality of the Serre spectral sequence,

Thus

$$
d_{l}(K)\left(p^{\gamma(K)} E_{2}^{0, *}(K)\right)=0 \text { for } 2 \leqq r \leqq k .
$$

$$
p^{r(K)} H^{*}(F ; Z)=p^{\gamma(K)} E_{2}^{0, *}(K) \subset E_{\infty}^{0, *}(K)=\operatorname{Im}\left(i_{K}^{*}\right) \subset H^{*}(F ; Z) .
$$

For each $K$, let $w_{k}=j_{K}^{*}\left(x_{k}\right)$, so that $i_{K}^{*}\left(w_{k}\right)=p^{\sigma_{k}} y_{k}$ and let $z_{k} \in$ $H^{k N}(\Omega X[K] ; Z)$ be such that $i_{K}^{*}\left(\boldsymbol{z}_{k}\right)=p^{\gamma(K)} y_{k}$, as guaranteed by Lemma 3.2. If $\sigma_{k}>\gamma(K)$ we have that

$$
w_{k}-p^{\sigma_{K}-\gamma(K)} z_{k} \in \operatorname{Ker}\left(i_{K}^{*}\right) .
$$

Lemma 3.3. If $N$ does not divide $K$, there exists an integer $M(K)$ such that $M(K) \operatorname{ker}\left(i_{K}^{*}\right)=0$.

Proof. In the Serre spectral sequence for $g_{K}, H^{l}(\Omega X[K] ; Z)$ is filtered by groups $D_{i, l-i}, 0 \leqq i \leqq K+1$, such that

(1) $D_{0, l}=H^{l}(\Omega X[K] ; Z)$,

(2) $D_{K+1, l-K-1}=\{0\}$,

and

(3) $D_{i, l-1} / D_{i+1, l-i-1} \approx E_{\infty}^{i, l-i}[K]$.

Hence $D_{1, l-1}=\operatorname{ker}\left(i_{K}^{*}: H^{l}(\Omega X[K] ; Z) \rightarrow H^{l}(F ; Z)\right)$. 
We can ensure that $D_{1, l-1}$ is a finite group by knowing that each $E_{2}^{i, l-i} \approx H^{i}\left(K\left(Z_{p}, N\right)^{(K)} ; H^{l-i}(F ; Z)\right)$ is finite for $i \geqq 0$. We have that $H^{i}\left(K\left(Z_{p}, N\right)^{(K)} ; Z\right)$ is finite except for $i=0$ or $K$. By condition (c) of the theorem $H^{j}(\Omega X ; Q)=0$ if $N$ does not divide $j$. Since $i^{*}$ : $H^{*}(\Omega X ; Q) \rightarrow H^{*}(F ; Q)$ is an isomorphism $E_{2}^{i, l-1}$ is finite for all $i \leqq 1$.

From now on we assume that $N$ does not divide $K$. Since $w_{k}$ $p^{\sigma_{k}-r(K)} z_{k} \in \operatorname{ker}\left(i_{K}^{*}\right), M(K)\left[w_{k}-p^{\sigma_{k}-\gamma(K)} z_{k}\right]=0$.

LemMA 3.4. If $g$ compresses into $K\left(Z_{p}, N\right)^{(K)}$, there exists a map $r_{K}: \Omega X \rightarrow \Omega X[K]$ such that $j_{K} r_{K} \sim 1_{\Omega X}$.

Proof. Immediate from the covering homotopy property (see [9] p. 689).

If $g$ were compressible into the $K$-skeleton of $K\left(Z_{p}, N\right)$ we could apply the map $r_{K}$ given by 3.4 to get

$$
M(K) x_{k}=M(K) p^{\sigma_{k-\gamma(K)}} r_{K}^{*}\left(z_{k}\right),
$$

which contradicts the hypothesis that each $x_{k}$ is a free generator of $H^{k N}(\Omega X ; Z)$.

Consequently the map $g: \Omega X \rightarrow K\left(Z_{p}, N\right)$ is not deformable into the $K$-skeleton of $K\left(Z_{p}, N\right)$ for any $K$ not divisible by $N$, hence not deformable into any finite skeleton. Since $g$ factors as $\Omega X \stackrel{\Omega f}{\rightarrow} \Omega Y \rightarrow$ $K\left(Z_{p}, N\right)$, the map $\Omega f$ is incompressible.

We proceed now to the proofs of Theorems 1.1 and 1.2. To show that $c(G, n) \leqq N$, for some $N$, we need to produce an $N$-dimensional space $X$ and a map $f: X \rightarrow K(G, n)$ such that $f$ is $\Omega$-incompressible. We shall take our spaces $X$ to be appropriate skeleta of our $K(G, n)$ 's.

Proof of Theorem 1.3. We apply 3.1 by letting $Y=K\left(Z_{p} k, 2 n\right)$, $N=2 n+2 p-2, X=K\left(Z_{p} k, 2 n\right)^{(N+1)}$, and $f: X \rightarrow Y$ be the inclusion

$$
f: K\left(Z_{p} k, 2 n\right)^{(N+1)} \subset K\left(Z_{p} k, 2 n\right) .
$$

If $l \leqq N+1, f^{*}: H^{l}(Y ; G) \rightarrow H^{l}(X ; G)$ is monic for any group $G$. Consequently $f^{*}\left(P^{1} \beta\left(\iota_{2 n}\right)\right)$ is a nonzero element of $H^{N+1}\left(X ; Z_{p}\right)$ that is in the kernel of $\beta$. (Here $P^{1}$ and $\beta$ denote the Steenrod operations.) Thus there exists a free generator $\bar{x}$ of $H^{N+1}(X ; Z)$ such that

$$
\rho_{p}(\bar{x})=f^{*}\left(P^{1} \beta\left(\iota_{2 n}\right)\right) .
$$

Set $x=\sigma^{*}(\bar{x})$. (Here $\sigma^{*}$ denotes the cohomology suspension.) Noting that $N$ is even and that in the Serre spectral sequence of the path space fibration of $X, d_{N+1}: E_{N+1}^{0 * *} \rightarrow E_{N+1}^{N+1, *}$ is an isomorphism, we see 
that $x$ generates a divided polynomial subalgebra of $H^{*}(\Omega X ; Z)$. Furthermore, if $\mu$ denotes that multiplication in $\Omega X$, then the $k$-fold interaction of $\mu^{*}$ contains a term

$$
\left(\mu^{*}\right)^{k}(x)=\cdots+k ! x \otimes \cdots \otimes x(k \text { factors })+\cdots
$$

and hence $x_{k}=(1 / k !) x^{k}$ is a free generator of $H^{k N}(\Omega X ; Z)$. Thus condition (a) of Theorem 3.1 is satisfied.

By the naturality of the Steenrod operations, the cohomology suspension, and reduction $\bmod p$, condition $(b)$ is satisfied. Condition (c) is obvious, since $X$ is the $(N+1)$-skeleton of a space $Y$ whose reduced rational cohomology is trivial.

Hence the hypotheses of Theorem 3.1 are satisfied and Theorem 1.3 is proven.

Proof of Theorem 1.4. Suppose $n>1$. We would like to apply 3.1 to $Y=K(Z, 2 n), N=2 n+8$, and $X=K(Z, 2 n)^{(N+1)}$, as in the proof of Theorem 1.3. However we would fail to satisfy condition (c). So instead we consider the composition

$$
K\left(Z_{2}, 2 n-1\right)^{(N+1)} \stackrel{h}{\longrightarrow} K(Z, 2 n)^{(N+1)} \stackrel{i}{\longrightarrow} K(Z, 2 n),
$$

where $h$ is a cellular map induced by a nontrivial map $K\left(Z_{2}, 2 n-1\right) \rightarrow$ $K(Z, 2 n)$. Then $h^{*}\left(S q^{6} S q^{3} \iota_{2 n}\right)=S q^{6} S q^{3} S q^{1}\left(\ell_{2 n-1}\right)$, and we let $f: X \rightarrow Y$ be

$$
i h: K\left(Z_{2}, 2 n-1\right)^{(N+1)} \rightarrow K(Z, 2 n)
$$

and take $\bar{x}$ to be a free generator that reduces to $S q^{6} S q^{3} S q^{1}\left(\ell_{2 n-1}\right)$. The proof then proceeds as that of Theorem 1.3.

4. Proof of Theorem 2.1. The connection between compressibility and Čech cohomology is made by the following result.

LEMma 4.1. If there exists an $\Omega$-incompressible map $f: X \rightarrow$ $K(G, n)(n>1)$, where $X$ is a finite complex, then $H_{f}^{n}(-; G)$ is not a homotopy functor on the category of countable CW-complexes.

Proof. Specifically, if $\bar{P} X$ denotes the Milnor universal bundle (path-space) on $X,[10]$, we shall show that $H_{f}^{n}(\bar{P} X ; G) \neq 0$. Let $\bar{\Omega} X$ denote the Milnor cellular loopspace of $X$. We have a diagram

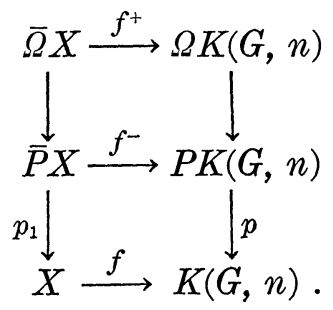


Since $f^{+}$is homotopy-equivalent to $\Omega f$, our hypothesis guarantees that $f^{+}$is not deformable into any compact subset of $\Omega K(G, n)$.

Now suppose that $H_{f}^{n}(\bar{P} X ; G)=0$. This means, according to 2.1, that $[\beta \bar{P} X ; K(G, n)]=0$. Let $i: \bar{P} X \rightarrow \beta \bar{P} X$ denote the inclusion. Since $X$ is compact, $p_{1}$ extends to $\hat{p}_{1}: \beta \bar{P} X \rightarrow X$ such that $\hat{p}_{1} i=p_{1}$. By our hypothesis, $f \hat{p}_{1} \sim *$. Hence there exists a lift $g: \beta \bar{P} X \rightarrow$ $P K(G, n)$ such that $p g=f \hat{p}_{1}$. Since $p g i=f \hat{p}_{1} i=f p_{1}, g i$ is a fiberpreserving map. Since $\bar{P} X$ is contractible, $g i \sim f^{+}$. Using again the contractibility of $\bar{P} X$ and the fact that $n>1, g i$ is vertically homotopic to $f^{+}$. Hence $f^{-}$is homotopic to $(g i) \mid \Omega X$, as maps $\bar{\Omega} X \rightarrow$ $\Omega K(G, n)$. Because $g i$ factors through $\beta \bar{P} X$ its image is contained in a compact subset. But that is a contradiction to the $\Omega$-incompressibility of $f$.

Proof of Theorem 2.1. In the proofs of Theorems 1.3 and 1.4, finite complexes were produced and maps to $\left(K\left(Z_{p} k, 2 n\right)(N \geqq 1)\right.$ and $K(Z, 2 n)(n>1)$, respectively that were $\Omega$-incompressible. By Lemma 4.1 , the functors $H^{2 n}\left(-; Z_{p} k\right)(n \geqq 1)$ and $H^{2 n}(-; Z)(n>1)$ do not satisfy the homotopy axiom on the category of countable CWcomplexes.

\section{REFERENCES}

1. A. Calder, Cohomology of finite covers, Trans. Amer. Math. Soc., 218 (1976), 349-352.

2. A. Calder and J. Siegel, Homotopy and extensions, Categorical Topology Mannheim 1975, Springer Lecture Notes 540 (1976).

3. Homotopy and uniform homotopy, Trans. Amer. Math. Soc., 235 (1978), $245-270$.

4. Kan extensions of homotopy functors, J. Pure Appl. Algebra, 12 (1978), 253269.

5. E. Čech, Theorie generale de l'homologie dans un espace quelconque, Fund. Math., 19 (1932), 149-183.

6. A. Dold, Lectures on Algebraic Topology, Springer-Verlag, Berlin-Heidelberg, 1972.

7. C. H. Dowker, Mapping theorems for noncompact spaces, Amer, J. Math., 69 (1947), 200-242.

8. S. Eilenberg and N. Steenrod, Foundations of Algebraic Topology, Princeton Univ. Press, Princeton N.J., 1952.

9. J. Goldfeather, Incompressibility and fibrations, Illinois J. Math., 21 (1977), 688-702. 10. J. Milnor, Construction of universal bundles I, Ann. of Math., 63 (1956), 272-284.

11. S. Weingram, On the incompressibility of certain maps, Ann. of Math., 93 (1971), 476-485.

Received May 16, 1980.

Birkbeck College

UNIVERSITY OF LONDON

London, EngLand

AND

New Mexico State University

Las Cruces, NM 88003 


\section{PACIFIC JOURNAL OF MATHEMATICS}

\section{EDITORS}

DONALD BABBITT (Managing Editor)

University of Galifornia

Los Angeles, California 90024

Hugo RossI

University of Utah

Salt Lake City, UT 84112

C. C. MOORE AND ANDREW OGG

University of California

Berkeley, CA 94720

\section{J. DugundjI}

Department of Mathematics University of Southern California

Los Angeles, California 90007

R. Finn AND J. Milgram

Stanford University

Stanford, California 94305

\section{ASSOCIATE EDITORS}

R. ARENS

E. F. BECKENBACH

B. H. NeUmanN

F. WOLF

K. YosHIDA

\section{SUPPORTING INSTITUTIONS}

UNIVERSITY OF ARIZONA

UNIVERSITY OF BRITISH COLUMBIA

CALIFORNIA INSTITUTE OF TECHNOLOGY

UNIVERSITY OF CALIFORNIA

MONTANA STATE UNIVERSITY

UNIVERSITY OF NEVADA, RENO

NEW MEXICO STATE UNIVERSITY

OREGON STATE UNIVERSITY
UNIVERSITY OF OREGON

UNIVERSITY OF SOUTHERN CALIFONIA

STANFORD UNIVERSITY

UNIVERSITY OF HAWAII

UNIVERSITY OF TOKYO

UNIVERSITY OF UTAH

WASHINGTON STATE UNIVERSITY

UNIVERSITY OF WASHINGTON 


\section{Pacific Journal of Mathematics}

\section{Vol. 94, No. 1 \\ May, 1981}

Willy Brandal, Conditions for being an FGC domain $\ldots \ldots \ldots \ldots \ldots \ldots \ldots$

Allan Calder and Frank Williams, Incompressibility of maps and the

homotopy invariance of Čech cohomology $\ldots \ldots \ldots \ldots \ldots \ldots \ldots \ldots \ldots$

Jacques Chaumat, Quelques propriétés du couple d'espaces vectoriels

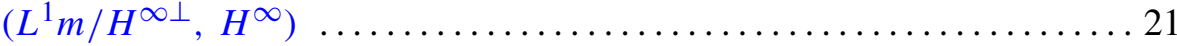

Manfred Droste and Rüdiger Göbel, Products of conjugate permutations . . 47

Jean Esterle, Rates of decrease of sequences of powers in commutative

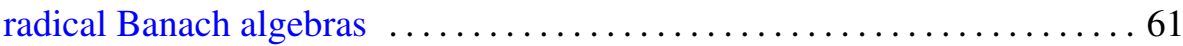

Allan Fryant, Ultraspherical expansions and pseudo analytic functions . . . 83

John Hannah, Homogenization of regular rings of bounded index. II . . . . . 107

Shigeru Haruki, On the theorem of S. Kakutani-M. Nagumo and J. L. Walsh

for the mean value property of harmonic and complex polynomials $\ldots .113$

Hugh M. Hilden, Representations of homology 3-spheres $\ldots \ldots \ldots \ldots \ldots 125$

Craig Huneke, A characterization of locally Macaulay completions . . . . . 131

Takesi Isiwata, Closed ultrafilters and realcompactness ................. 139

Joseph Weston Kitchen, Jr. and David A. Robbins, Tensor products of

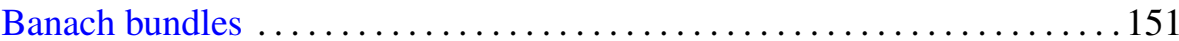

Allan J. Kroopnick, Note on bounded $L^{p}$-solutions of a generalized Liénard

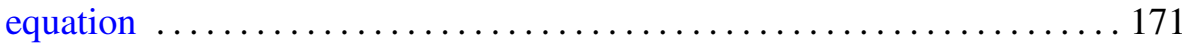

Ajay Kumar and Ajit Kaur Chilana, Spectral synthesis in products and quotients of hypergroups

Charles Livingston, Homology cobordisms of 3-manifolds, knot

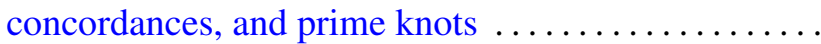

Hans Opolka, Projective representations of finite groups in cyclotomic fields

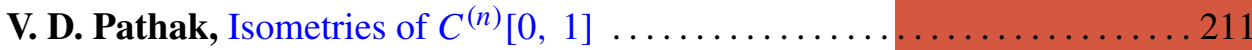

Mark Allan Pinsky, On the spectrum of Cartan-Hadamard manifolds . . . . . 2223

Judith Roitman, The number of automorphisms of an atomic Boolean

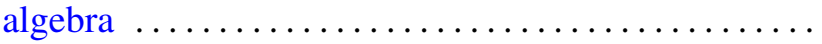

Kai Wang, Locally smooth torus group actions on integral cohomology complex projective spaces 